\title{
Modelling, measurement and optimization of self-noise of hydrophone with preamplifier
}

\author{
Yi Chen", Lisheng Zhou, Xiyang Guo, Tao He, and Jun Zhang \\ Hangzhou Applied Acoustics Research Institute, 311400, P. R. China
}

\begin{abstract}
Self-noise is an important specification of hydrophone with preamplifier. This paper presents the modelling, measurement and optimization of the self-noise of hydrophone with preamplifier. Through analyzing various noise sources of hydrophone and its preamplifier, an effective self-noise model of hydrophone with preamplifier was established, and the self-noise was mainly affected by the hydrophone quality factor, preamplifier noise and thermal noise of preamplifier input resistance. In order to measure the self-noise of hydrophone with preamplifier, a measurement system was constructed and used for measuring the self-noise of a RHSA30 hydrophone which manufactured by Hangzhou Applied Acoustics Research Institute (HAARI) and has a typical sensitivity of $-173 \mathrm{~dB}$. After optimization of the self-noise of RHSA30 hydrophone, the measured equivalent noise pressure spectral density level was close to $30 \mathrm{~dB}$ at the frequency of $1000 \mathrm{~Hz}$. The measurement results proved that the preamplifier current noise which mainly coming from the Operational Amplifier played a leading role at low frequencies of hydrophone with preamplifier. And some basic principles for self-noise optimization of hydrophone with preamplifier were given at the end.
\end{abstract}

\section{Introduction}

Hydrophones are electroacoustic transducers that produce electrical signals in response to water borne pressure signals [1], they were widely used in SONAR equipments and marine acoustic instruments for detecting underwater targets or measuring underwater sound signals. Hydrophone types include reference hydrophones and measuring hydrophones. Usually, reference hydrophones did not equip with integral preamplifiers, and they were calibrated by absolute calibration methods. Measuring hydrophones were often calibrated by comparison methods, and used for measuring the sound pressure in water directly. In order to increase Signal to Noise Ratio (SNR) of the measurement system, most of the measuring hydrophones were equipped with integral preamplifiers.

Generally, the hydrophones used in underwater acoustics were piezoelectric type, and the preamplifiers used in measuring hydrophones were voltage preamplifiers. The performance of underwater acoustical measurement systems were largely determined by the characteristic of measuring hydrophones with preamplifier, and self-noise is one of the important specifications for them, especially for the systems which measuring noises in water. It directly affects the equivalent noise pressure spectral density level and dynamic range of the underwater acoustical measurement system.

Much attention [2-5] had been paid to modelling, measurement and optimization of self-noise of hydrophones with preamplifiers in the past. Tims [3] studied the hydrophone preamplifier optimization in 1978, and proposed an equivalent circuit noise model including acoustic sensor, coupling network and preamplifier input stage for predicting the self-noise of hydrophone with preamplifier. Brown and Tims [4] continued the optimization work, they studied the hybrid microelectronics for low-noise hydrophones, and presented a DC and low-frequency AC analysis of a lownoise preamplifier design in 1987. In 1993, Straw [5] developed a noise prediction model of hydrophone preamplifier system, which could be used for calculating the electronics noise of preamplifier and hydrophone. With the development of electronics technology, Operational Amplifiers had been widely used in the integral preamplifiers of hydrophones, and related research work [6] of optimization of self-noise had been studied in Hangzhou Applied Acoustics Research Institute (HAARI) since 2012.

This paper presents the modelling, measurement and optimization of self-noise of hydrophone with preamplifier which using Operational Amplifier. Utilizing the equivalent circuit model analytical method, a model for evaluating noise level of piezoelectric hydrophone with preamplifier was established, and a system for measuring the self-noise of hydrophone with preamplifier was constructed. On the basis of these, the parameters of hydrophone with preamplifier such as capacitance and sensitivity of hydrophone, input resistor, preamplifier current noise and preamplifier voltage noise were investigated, and several basic principles for optimization of the self-noise of hydrophone with preamplifier were given at the end.

\footnotetext{
*Corresponding author: y.chen@163.com
} 


\section{Modelling of self-noise of hydrophone with preamplifier}

In the absence of sound pressure, noise and other interference, the noise pressure presenting at the electrical terminals was named as equivalent noise pressure of hydrophone, which is one of the important specifications used for evaluating the performance of hydrophone, and directly affect the SNR of the output signal of hydrophone. The preamplifier was the first circuit for the output open-circuit voltage of hydrophone which amplifying the output voltage signal of the hydrophone and realizing the transform of impedance. But noise was introduced when amplifying the signal, which including voltage noise and current noise. And the equivalent noise pressure was the combination of thermal noise contribution of sensor element and electronic noise of preamplifier. Usually, electronic noise was higher than thermal noise.

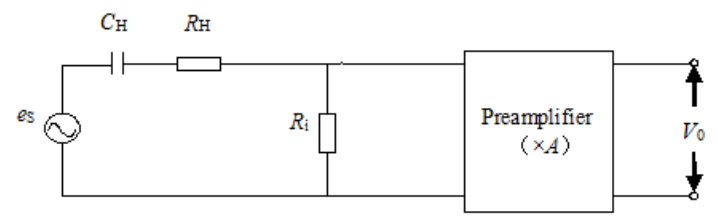

Fig. 1. Equivalent circuit of hydrophone with preamplifier.

Fig.1 shows the equivalent circuit of hydrophone with preamplifier. Hydrophone was represented by capacitance $C_{\mathrm{H}}$ in series with resistance $R_{\mathrm{H}}, e_{\mathrm{S}}$ represented the signal produced by sensor element, A represented the amplifying number of the preamplifier, $\mathrm{Ri}$ represented the input resistance of the preamplifier. Resistances of circuit would produce thermal noise, and its power spectral density could be written as

$$
e_{\mathrm{R}}^{2}=4 k T R
$$

where, $k$ was the Boltzmann's constant, which equals to $1.38 \times 10^{-23} \mathrm{~J} / \mathrm{K}$. $T$ was the temperature on conductor, $\mathrm{K}$. $R$ was the resistance or the real part of the impedance of conductor, $\Omega$.

Considering the noises produced by hydrophone and preamplifier, the self-noise model of hydrophone with preamplifier which shown in Fig. 2 could be got. In Fig.2, $e_{\mathrm{H}}$ was the thermal noise produced by $R_{\mathrm{H}}, e_{\mathrm{Ri}}$ was the thermal noise produce by $R_{\mathrm{i}}, e_{\mathrm{A}}$ was the preamplifier voltage noise, and $i_{\mathrm{A}}$ was preamplifier current noise. These noises would produce voltage noises at the output terminal of the preamplifier.

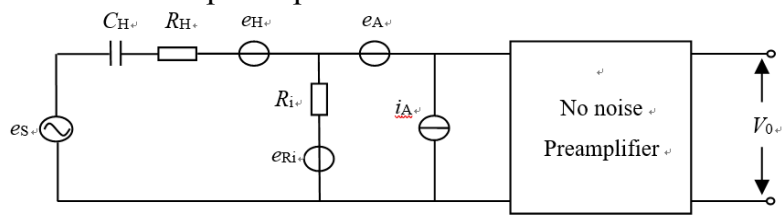

Fig. 2. Noise model of hydrophone with preamplifier.

Although the voltage noise and current noise sources of preamplifier were sometimes correlated, assuming that they were not so would result in a little or no error [5]. If the sensitivity of hydrophone was $M$, the equivalent noise sound pressure presenting at the electrical terminal of hydrophone with preamplifier could be written as

$$
P_{\mathrm{IN}}=\sqrt{\frac{4 K T \tan \delta}{\omega C_{\mathrm{H}} M^{2}}+\frac{1}{M^{2}}\left(e_{\mathrm{A}}^{2}+\frac{R_{\mathrm{i}}^{2} i_{\mathrm{A}}^{2}}{1+\omega^{2} R_{\mathrm{i}}^{2}\left(C_{\mathrm{Ci}}+C_{\mathrm{H}}\right)^{2}}+\frac{4 K T R_{\mathrm{i}}}{1+\omega^{2} R_{\mathrm{i}}^{2}\left(C_{\mathrm{Ci}}+C_{\mathrm{H}}\right)^{2}}\right)}
$$

where, the first term in equation (2) was the thermal noise due to hydrophone internal losses, and was independent of any cable or preamplifier parameters. This noise was the lowest limitation that could be achieved by the system of hydrophone with preamplifier. The second term in equation (2) was preamplifier noise, including preamplifier voltage noise, preamplifier current noise and preamplifier input resistance thermal noise.

Obviously, in order to get the lowest self-noise of hydrophone with preamplifier, the second term had better to be zero or as small as possible.

\section{Analysis of self-noise model of hydrophone with preamplifier}

\subsection{Impact of hydrophone quality factor}

The hydrophone quality factor $H$ equals to the square of hydrophone sensitivity $M^{2}$ plus hydrophone capacitance $C_{\mathrm{H}}$, that means

$$
H=M^{2} C_{\mathrm{H}}
$$

It could be seen from the equation (2), that with the increasing of hydrophone quality factor, the equivalent noise sound pressure would decrease. Although the values of $M$ and $C_{\mathrm{H}}$ were associated with series or parallel ways which had been used when hydrophone was constructed of several sensor elements, the hydrophone quality factor was constant if volume of sensor elements was not changing, and had nothing to do with the connection of elements.

\subsection{Analysis of preamplifier circuit}

As shown in Fig.3, the circuit of a preamplifier was designed and produced to study the impact of preamplifier noise. Where, OP was an integrated Operational Amplifier, $R_{1}$ and $R_{\mathrm{f}}$ constituted a negative feedback with its gain factor of $A=R_{1} / R_{\mathrm{f}}, C_{1}$ and $R_{\text {in }}$ constituted the preamplifier input stage, $C_{3}$ and $R_{\mathrm{L}}$ constituted the preamplifier output stage, and $R_{2}$ and $C_{2}$ were used for balancing the forward and backward direction impedance, which could also decrease the effect of bias current.

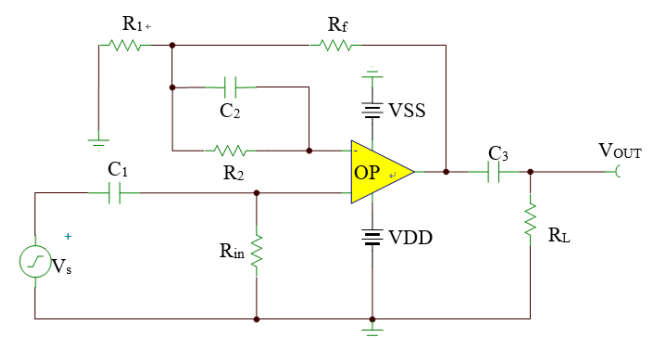

Fig. 3. Circuit diagram of preamplifier. 
Through the study of circuit simulation and experiment of actual hydrophone preamplifier noise optimization, it was found that

(1) Preamplifier current noise mainly came from Operational Amplifier, and preamplifier voltage noise not only came from the voltage noise of Operational Amplifier, but also came from the thermal noise of circuit resistances. Usually, current noise played a leading role at low frequency band, and it would be voltage noise when the frequency was above $1 \mathrm{kHz}$.

(2) The effect of thermal noise would decline through decreasing preamplifier circuit resistance. But the power consumed would be magnified at the same time, and the matters of heating and high power consumption, etc would be introduced. And these related factors should be considered when selecting the resistances of preamplifier circuit.

(3) Increasing the preamplifier input resistance would not magnify the noise of system; on the contrary, it would decrease the input resistance thermal noise impact on the system. In addition, the preamplifier input resistance should be high enough if hydrophone with preamplifier needed to work at low frequency band.

\section{Measurement of self-noise of hydrophone with preamplifier}

\subsection{A self-noise measurement system}

In IEC 60500:2017, the parameters used for evaluating the self-noise of hydrophone were equivalent bandwidth noise pressure and equivalent noise pressure spectral density. The equivalent bandwidth noise pressure was defined as a radio of noise voltage at a specified central frequency in the relevant frequency band present at the electrical terminals of the hydrophone, in the absence of sound pressure, noise and other interference, to its sensitivity at a specified frequency. It would be equivalent noise pressure spectral density when the bandwidth equals to $1 \mathrm{~Hz}$.

In order to measurement the equivalent bandwidth noise pressure or equivalent noise pressure spectral density of hydrophone with preamplifier, a self-noise measurement system was established [7]. Fig.4 shows the diagram of the self-noise measurement system. A hydrophone with preamplifier was put into a closed stainless steel barrel with its dimension of $400 \mathrm{~mm} \times$ $\phi 300 \mathrm{~mm}$ and wall thickness of $20 \mathrm{~mm}$. Through a hose connected to the vent on the lid, the barrel was vacuumized by a vacuum pump. In order to isolate vibration and absorb the sound, vibration isolators at the bottom of barrel and multilayer acoustical foam cotton in the barrel were further used. The output noise voltage of hydrophone with preamplifier was sampled by an NIPXI acquisition system with a PXIe-4498 dynamic signal acquisition card of very low voltage noise (7nV/ $\sqrt{\mathrm{Hz}} @ 1 \mathrm{kHz})$.

The practical testing proved that the chamber had a good isolation of noise for frequency above $100 \mathrm{~Hz}$, and relatively bad for frequency below $100 \mathrm{~Hz}$, and also had some electromagnetic shielding effect, especially at the high frequency band. Because the outside interference could not be fully isolated, the measurement results needed to be determined according to the actual situation to eliminate the invalid measurements.

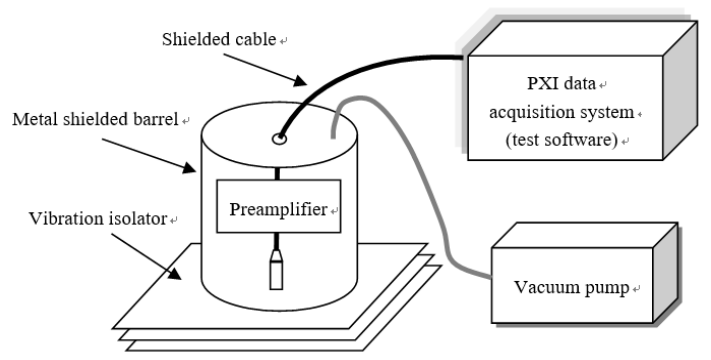

Fig. 4. Diagram of noise measurement system.

\subsection{RHSA30 hydrophone and its measurement results}

The hydrophone with preamplifier to be measured was a RHSA30 hydrophone which manufactured by HARRI, China. Its sensitive element was a piezoelectric ceramic sphere with a diameter of $30 \mathrm{~mm}$, and it had an integral preamplifier and $15 \mathrm{~m}$ long integral cable. The typical sensitivity of RHSA30 was $-173 \mathrm{~dB}$ with $20 \mathrm{~dB}$ preamplifier gain.

The results of equivalent noise voltage spectral density level measured at the electrical terminal of RHSA30 hydrophone was shown in Fig.5.

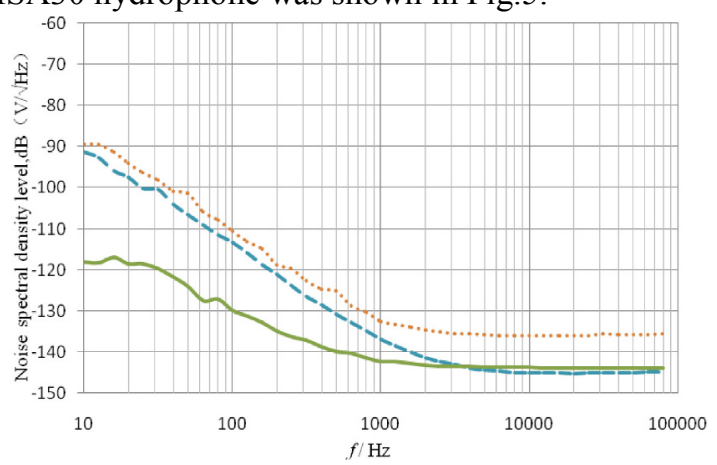

Fig. 5. Noise spectral density measured at the electrical terminal of RHSA30 hydrophone.

In Fig.5, the red dot line was the equivalent noise spectral level measured before optimization. The blue dash line was the equivalent noise spectral level measured after optimization of the feedback impedance which was one tenth of the equivalent noise before optimization. The green line was the equivalent noise spectral level measured after further optimization of the Operational Amplifier. It was obvious that the self-noise of hydrophone with preamplifier was greatly improved at low frequency band, although it was a little worse at high frequency band. At the frequency of $1000 \mathrm{~Hz}$, the equivalent noise pressure spectral level of RHSA30 hydrophone was close to $30 \mathrm{~dB}$, which is much lower than Seastate Zero.

The self-noise of two Operational Amplifiers which applied in RHS30 hydrophone were shown in Fig.6. Where, the line in blue was the self-noise of the old Operational Amplifier, and the dot line in red was the self-noise of the new Operational Amplifier. Comparing 
the equivalent noise spectral density level of hydrophone shown in Fig.5 to the self-noise of two Operational Amplifiers shown in Fig.6, it was found that these two results were very similar in the direction of the curves, with only some minor difference at low frequency band close to $10 \mathrm{~Hz}$. It also proves the correctness of the measurement results of the equivalent noise spectral density of RHSA30 hydrophone.

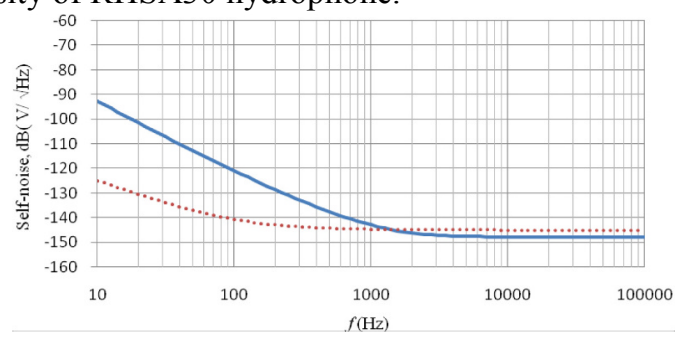

Fig. 6. Self-noise of two Operational Amplifiers.

\section{Optimization principles for hydrophone with preamplifier}

From the above discussion, some useful results for noise optimization of hydrophone with preamplifier could be concluded as follows.

Firstly, the noise optimization of hydrophone with preamplifier should be considered according to the noise situation where you would use it. Only if the equivalent noise pressure of measurement system is higher than the circumstance noise, the self-noise of hydrophone with preamplifier should be optimized to assure the performance of the measurement system.

Secondly, the noises of hydrophone with preamplifier mainly came from preamplifier circuits, selection of Operating Amplifier, feedback resistance and input resistor are very important to decrease the self-noise level, especially the Operating Amplifier.

Thirdly, the noise of hydrophone with preamplifier could be effectively decreased through increasing the hydrophone quality factor, which could be improved by enlarging the volume of sensor element or using piezoelectric material with better performance.

\section{Conclusions}

The first stage work for optimization of hydrophone with preamplier had achieved a significant result, and the next work is to improve the measurement condition to measure and further optimize the equivalent noise pressure spectral density of the hydrophone with preamplifier.

This work was funded by Chinese National Key Research and Development Program 2016YFF0200900.

\section{References}

1. IEC 60500. Underwater Acoustics - Hydrophones Properties of hydrophones in the frequency range 1 $\mathrm{Hz}$ to $500 \mathrm{kHz}$ (International Electrotechnical Commission, Geneva, 2017)
2. J. W. Young, J. Acoust. Soc. Am., 61, 1471-1476 (1977)

3. A. C. Tims, Hydrophone preamplifier optimization - Prediction of hydrophone self-noise by a noise model. Naval Research Laboratory, Washington, D.C. (1978)

4. C. K. Brown and A. C. Tims, Hydrophone reamplifier ptimization - Hybrid microelectronics for low-noise hydrophones. Naval Research Laboratory, Washington, D.C. (1987)

5. T B Straw, Noise prediction for hydrophone/ preamplifier systems. Naval Undersea Warfare Center Detachment, New London (1993)

6. Xiyang Guo, Research on self-noise characteristics of hydrophone and preamplifier, Master thesis of Hangzhou Applied Acoustics Research Institute (2013)

7. Yi Chen, Han Zhao, Wenjun Yuan, Measurements of underwater electroacoustic parameters (Ordnance Industry Publishing House, Beijing, 2017) 